\title{
Laparoscopic Hernia Repair and Its Validation by Second-Look Inspection to Internal Inguinal Rings in Children with Patent Processus Vaginalis
}

\author{
Masao Endo, Michinobu Ohno, Fumiko Yoshida, \\ Miwako Nakano, Toshihiko Watanabe and Etsuji Ukiyama \\ Department of Pediatric Surgery, Saitama City Hospital \\ Japan
}

\section{Introduction}

We developed a unique technique for achieving the completely extraperitoneal ligation of a patent processus vaginalis (PPV) without skipping any areas (Endo et al., 2001). This technique has been used for the treatment of more than 1,600 children. A previous comparative study of this technique with the traditional cut-down repair method proved the superiority of this technique with respect to the parental perspective and choice, operative time, recurrence rate, metachronous appearance of contralateral hernia, complication of the reproductive system, and cosmetic results (Endo et al., 2009).

However, some concerns have been voiced regarding the use of laparoscopic herniorrhaphy in children such as the use of a simple closure without the division of the hernial sac, evidence of a completely closed internal inguinal ring (IIR), validity with regard to future recurrence, and the high risk of adhesion (Miltenburg et al., 1998, Gorsler et al., 2003, Saranga et al., 2008).

Since 1996, when we began to close PPV laparoscopically, we have conducted prospective studies involving laparoscopic inspection at previous operation sites at every opportunity so as to validate the efficacy of this procedure. The purpose of this paper was to introduce our procedure and the use of recently devised, innovative "Endoneedle kit", comparing the outcome, including the morphological appearance of the IIRs in second-look operations, with the outcome of traditional cut-down herniorrhaphy performed during the same period.

\section{Materials and methods}

[Operative procedures] The kit consists of a $70 \mathrm{~mm}$ needle covered with a 17-G metal sheath (puncture needle), an 18-G dull-tipped needle with a wire loop inside the barrel for the internal introduction of a 2-0 suture used to send the suture (suture sender), and a 20-G dull-tipped needle with a wire loop inside the barrel used to retrieve the suture (suture retriever)(Fig.1). These needles are packaged together and provided as a sterilized, nonreusable package. 


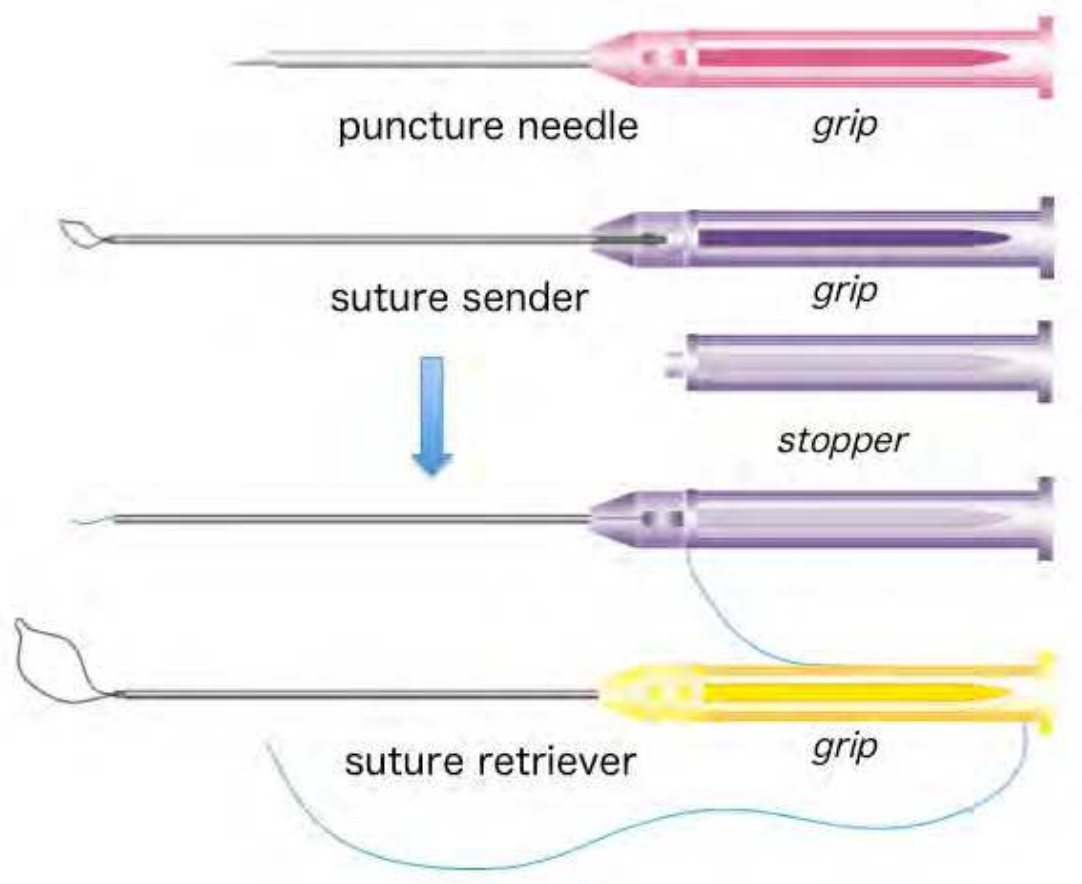

Fig. 1. Composition of the Endoneedle Kit. The needle contained inside the puncture needle can be released by twisting and pulling the grip. The suture (0-2 non-absorbable twisted suture) is pulled through the barrel of the suture sender and fixed with a stopper. The suture is picked up by a grasper through the loop of the suture retriever and caught by pulling back the grip.

The puncture needle is inserted percutaneously and advanced along the lower half of the IIR extraperitoneally across the cord and vessels and protruded into the peritoneal space at the opposite side of the puncture site, then withdrawn leaving the metal sheath in place. A 2-0 suture is then delivered into the peritoneal space through the metal sheath using the suture sender and placed at the site by withdrawing the sheath and the suture sender. The puncture needle is then advanced along the upper half of the IIR and protruded into the peritoneal space at the site where the previously sent suture has penetrated the peritoneum. The suture is then retrieved by the suture retriever passed through the metal sheath of the puncture needle and tied extracorporeally, enabling a complete extraperitoneal ligation of the ring (Fig.2).

[Study design] A consecutive series of 1,943 children who underwent definitive herniorrhaphy between 1996 and 2009 was analyzed. At the preoperative guidance session, three methods were proposed to the patients' parents (Fig.3): a traditional cut-down repair (CDR) for the affected side only (CDR-A), an additional diagnostic laparoscopy for contralateral IIR inspection with simultaneous closure of the contralateral PPV (cPPV) $(\mathrm{CDR}+\mathrm{L})$, and a laparoscopic completely extraperitoneal PPV closure with routine closure of the cPPV during the same procedure (LCEPC). Either a CDR-A, CDR+L or LCEPC was selected according to the parent's preference and with their informed consent. 


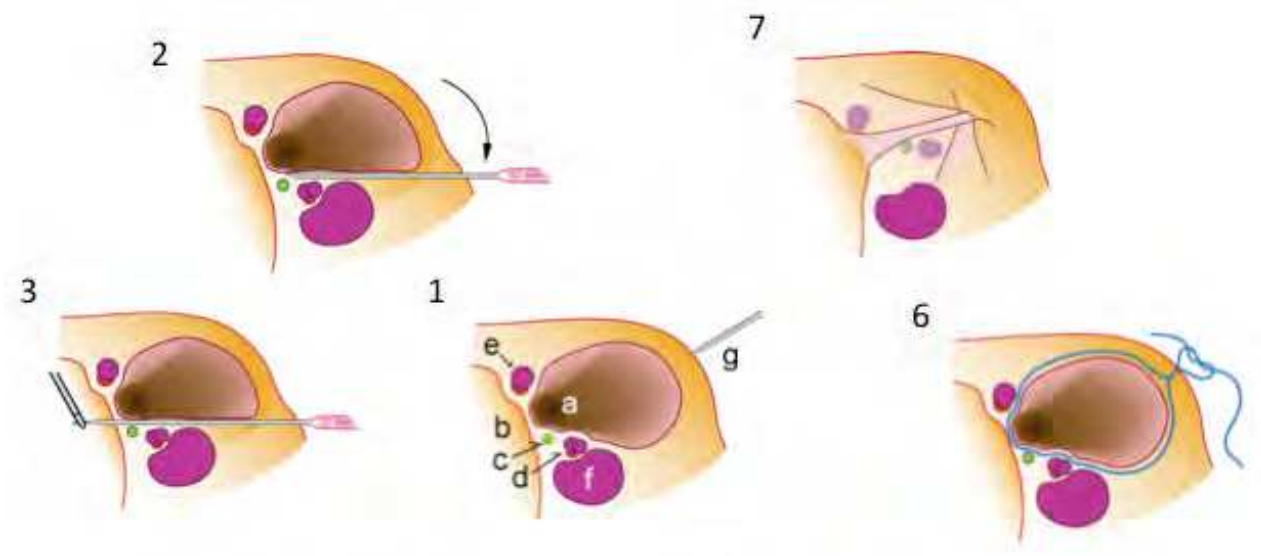

4

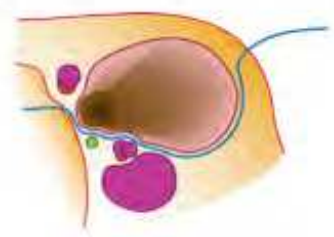

5

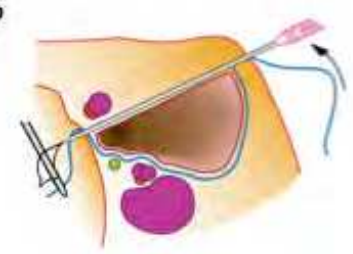

Fig. 2. Schematic drawing of completely extraperitoneal closure of the right-sided PPV. 1. Anatomy of male IIR. a, orifice of PPV; b, wall of pelvic pouch; c, spermatic duct; $\mathrm{d}$, testicular vessels; $\mathrm{e}$, inferior epigastric vessels; $\mathrm{f}$, external iliac vein, $\mathrm{g}$, puncture needle being inserted percutaneously. 2. The puncture needle goes extraperitoneally across the common iliac vein, testicular vessels and spermatic cord, which are easily facilitated by pressing the needle down. 3. A suture is carried into the peritoneal cavity by the suture sender and picked up by a grasper. 4 . The suture is left along the lower half of the IIR. 5 . The free end of the suture is picked up by the suture retriever inserted through the puncture needle that has been placed along the upper half of the IIR. 6. The orifice of the PPV has been encircled without any skip area. 7 . The IIR has been tied up, while the spermatic cord and the testicular vessels are drawn close to the ligation but spared. 


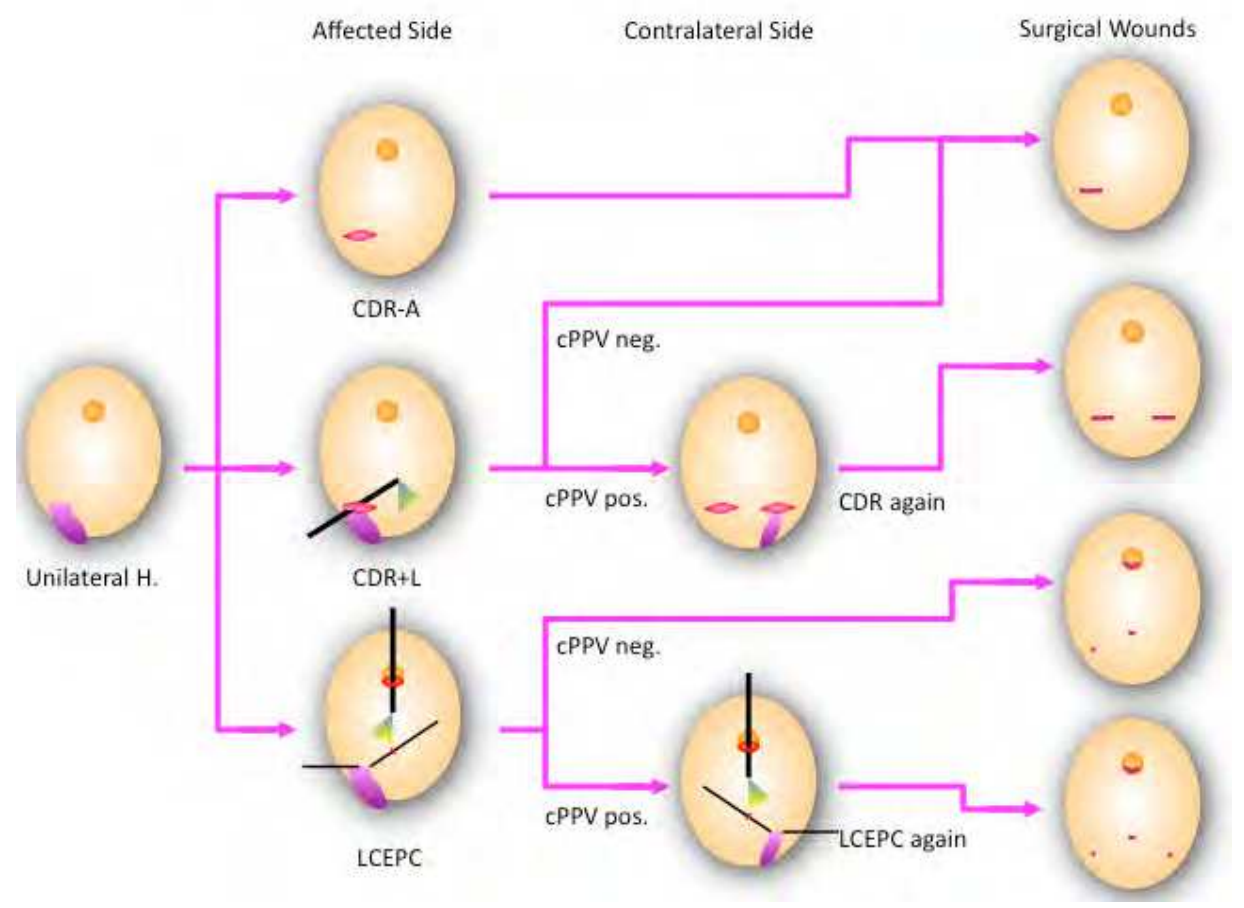

Fig. 3. Instruction for parental perspective and choice. There are three choices; 1 , cut-down repair for affected side only (CDR-A); 2, CDR with diagnostic laparoscopy with CDR again for $\mathrm{CPPV}$ if present $(\mathrm{CDR}+\mathrm{L})$; 3 , laparoscopic completely extraperitoneal PPV closure with simultaneous closure for CPPV (LCEPC).

The LCEPC and CDR groups were then compared according to parental selection, distribution of sex, age, clinical lateralities, associated morbidities, operation times, development of contralateral hernia, recurrence and major complications. The patients were followed up regularly in our outpatient clinic for 7 months after the operation, and during visits for any other complaints or morbidities thereafter. The follow-up period ranged from one to 14 years.

Among these children, the IIRs of 58 children who had undergone a second laparoscopic operation were investigated with regard to the morphological appearance of the IIRs during the revisit, with special reference to the predicted future outcome. The distributions of the primary operations of these 58 children and the reasons for the second operation are showed in Figure 4.

Based on the procedure used for the primary operation, the IIRs were classified into a postcut-down repair group (post-CDR) and a post-laparoscopic repair group (post-LCEPC). Among the IIRs that were inspected, those found to be closed (closed IIR = C-IIR) were investigated in terms of their morphological variations: from a flat scar to degeneration with a dimple, cicatricial tissue gathering, or adhesion. The spectra of the morphological deviations were compared between the post-CDR and post-LCEPC groups.

[Statistical analysis] Continuous data were expressed as the mean +/- standard deviation (SD). Statistical significance was calculated using a two-tailed $t$-test. For proportional data, the chi-square test was used. 


\section{Results}

[Results for definitive herniorrhaphy]

Parents showed a greater preference for LCEPC, accounting for 1,631 children (86\%) compared with 312 children who underwent CDR with or without a diagnostic laparoscopy (CDR-A, 66; CDR+L, 246) (Fig. 5).

\section{Primary operation Reasons for second-look operation}

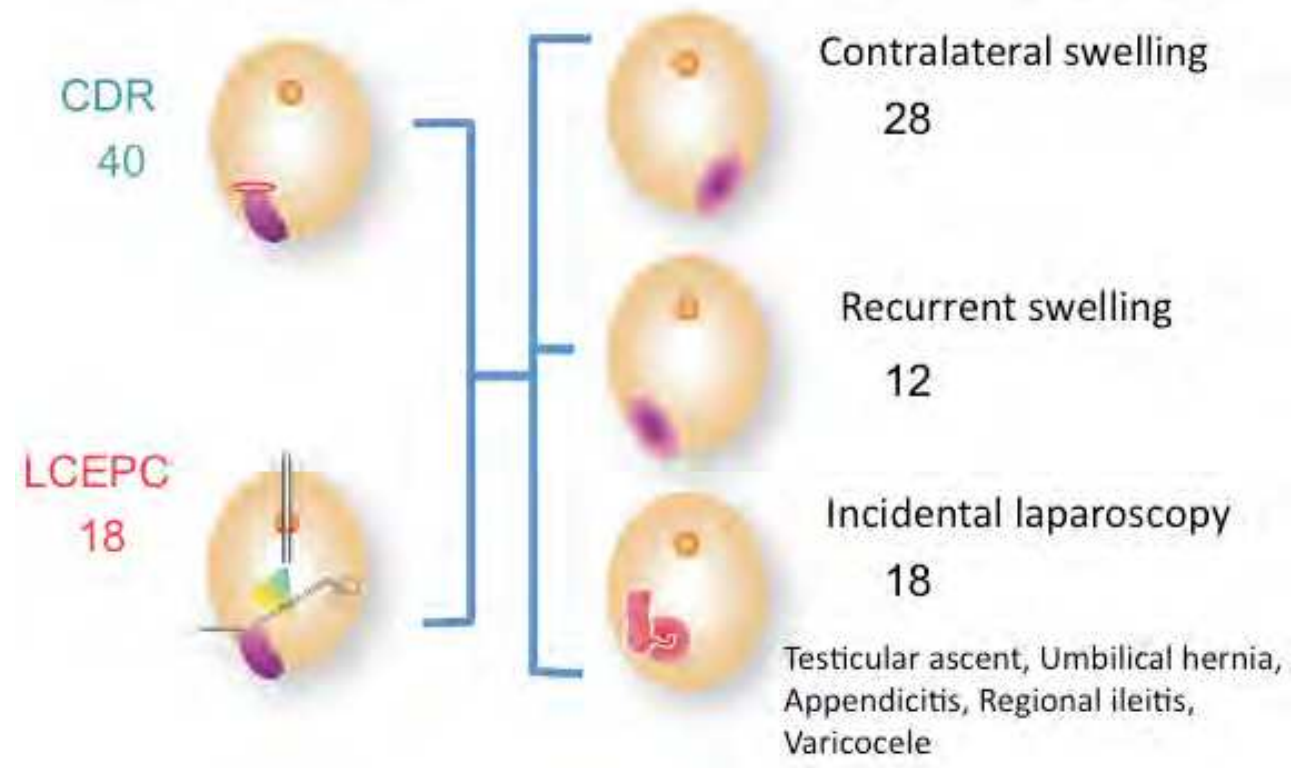

Fig. 4. Distributions of primary operation and reasons for second-look operation. Incidental laparoscopies were performed during treatment for testicular ascent (8), umbilical hernia (4), appendicitis (2), regional ileitis (1), varicocele (1) and miscellaneous (2). Numbers in parenthesis indicate numbers of patient. 


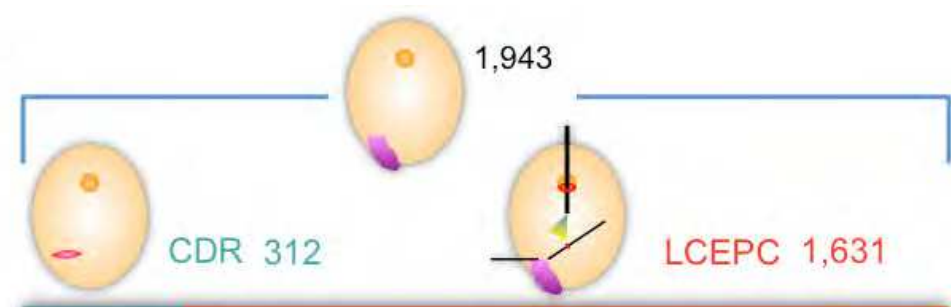

\section{$16 \%$}

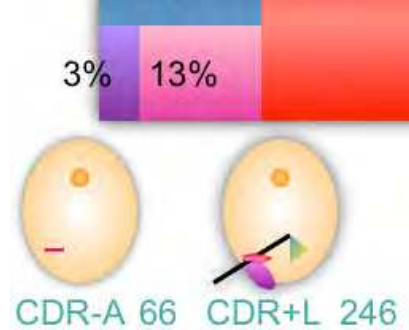

$86 \%$

Fig. 5. Patients who underwent chosen procedures. Total number of patients was 1,943; Number of patients in LCEPC group was 1,631 (86\%) and in CDR was $312(16 \%)$, in which CDR-A was 66 (3\%) and CDR+L was $246(13 \%)$.

The age distributions for the CDR and LCEPC groups (around 3.9 and 3.7 years, respectively) were not significantly different (Fig. 6).

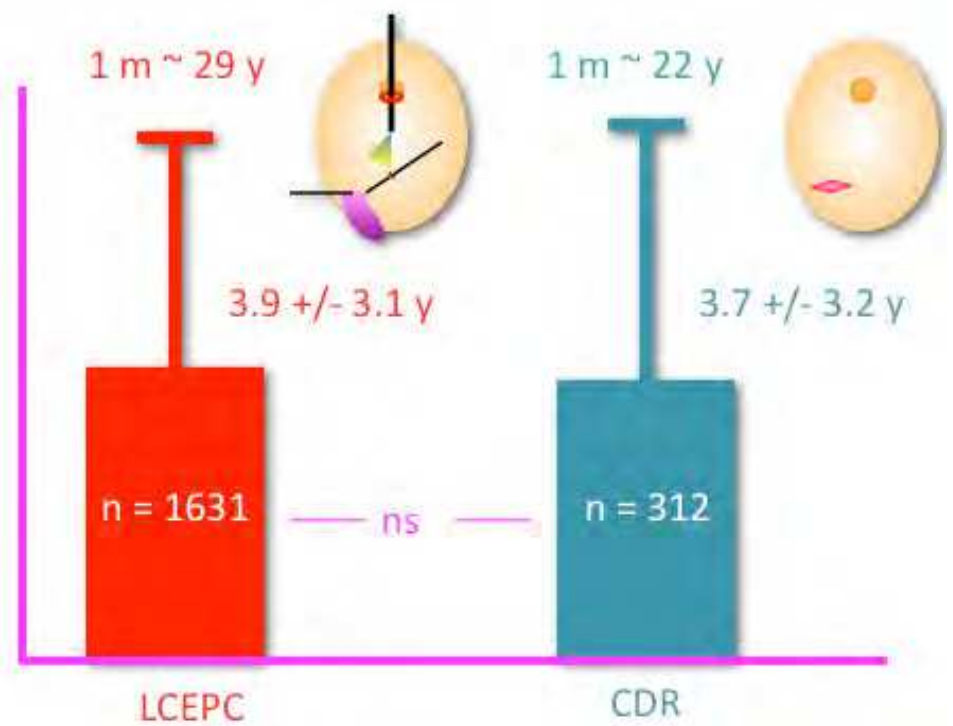

Fig. 6. Distribution of ages. Data are shown in mean +/- standard deviation. ns, statistically not significant. 
The distribution of clinical hernia sides was also the same for both groups (Fig. 7).
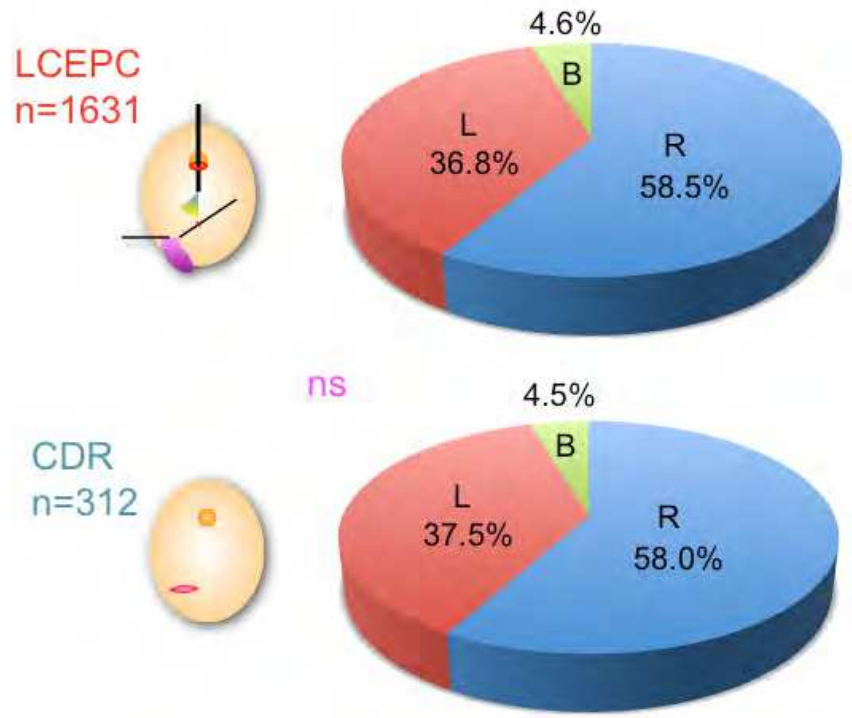

Fig. 7. Distributions of lateralities in clinical hernia. Differences between both groups were not statistically significant.

On the other hand, the sex distribution showed a female predominance in the LCEPC group because of the parents' perspective regarding the cosmetic superiority of laparoscopic repair (Fig. 8).
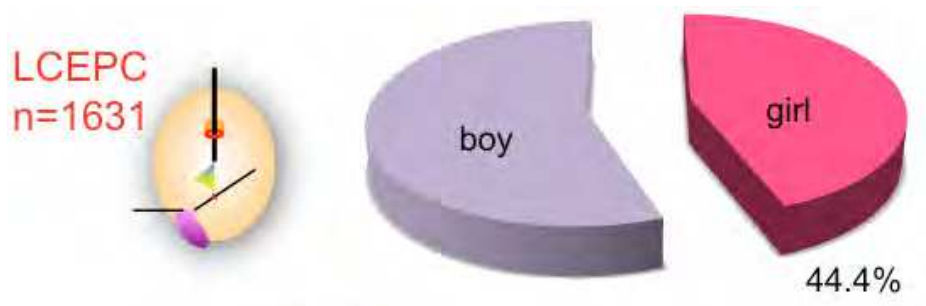

\section{$P<0.001$}
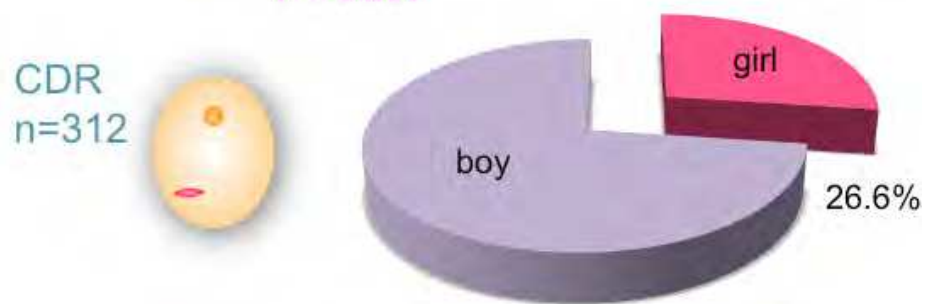

Fig. 8. Sex distribution. LCEPC group included more girls (44.4\%) than CDR group (26.6\%), $\mathrm{p}<0.001$. 
Regarding the distributions of associated morbidities necessitating a combined operation, no statistically significant differences were observed between the two groups with the exception of umbilical hernia/cysts. Umbilical deformities were seen 4 times more frequently in the LCEPC group because of the parental desire for an umbilicoplasty during the same session (Fig. 9).

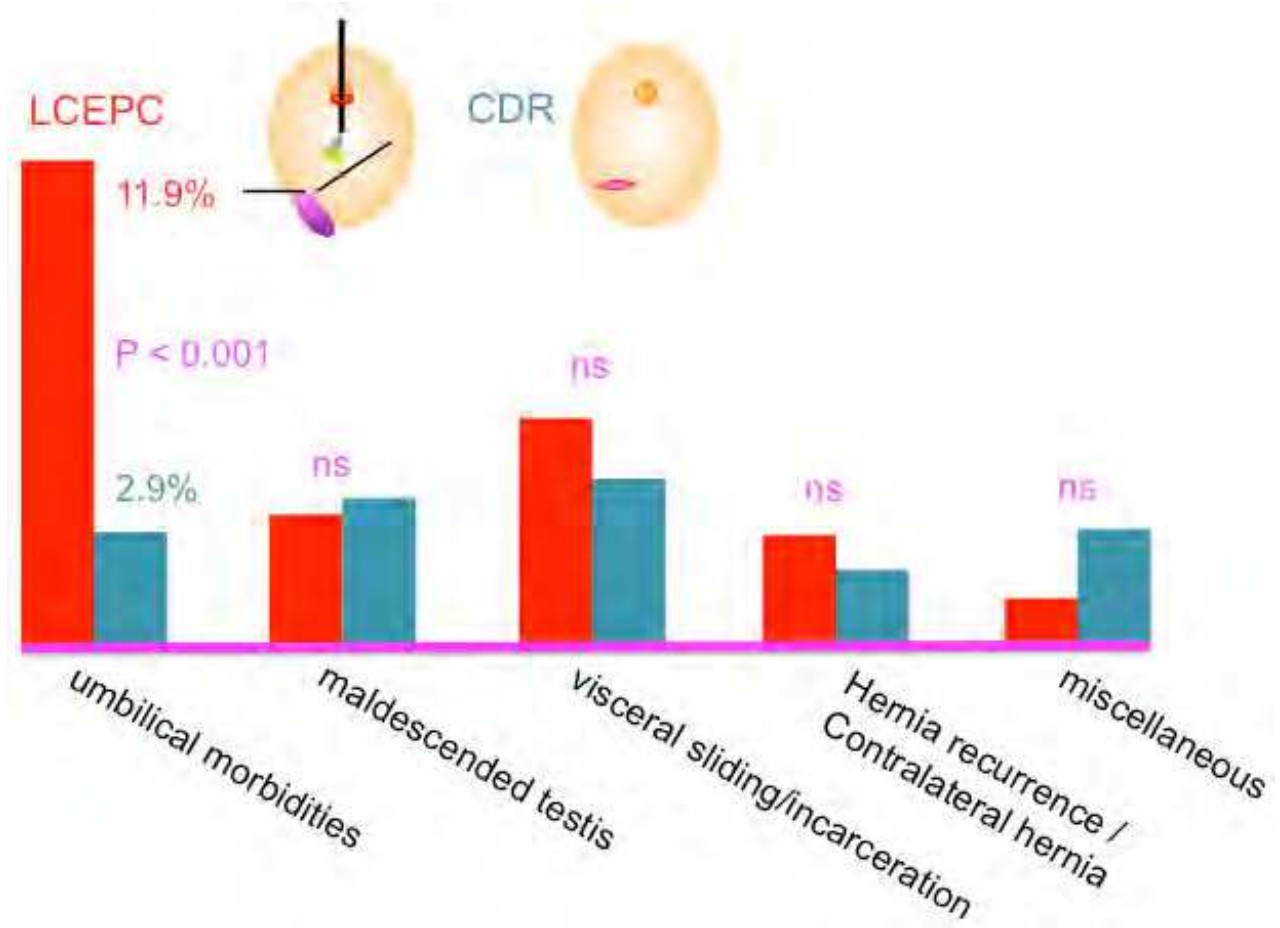

Fig. 9. Associated morbidities necessitating a combined operation. There were no statistically significant differences in the distributions of associated morbidities except umbilical morbidities such as hernia, cystic degeneration or ugly-looking umbilicus (LCEPC, 194, 11.9\% vs. CDR, 9, 2.9\%, p<0.001).

The mean operation times for unilateral repair were the same in the LCEPC and CDR groups, while the mean operation time for bilateral repair was 11 minutes shorter in the LCEPC group ( $\mathrm{p}<0.001)$ (Fig. 10).

Intra- and post-operative complications are listed in Table 1. A statistically significant difference was observed in the incidence of intra-operative injuries to the reproductive system between the LCEPC and CDR groups ( $0 \%$ vs. $0.6 \%, p<0,001)$. The incidence of postoperative contralateral hernia was lower in the LCEPC group, but the difference was not statistically significant. Postoperative hernia recurrence was significantly lower in the LCEPC group $(\mathrm{p}<0.001)$. 


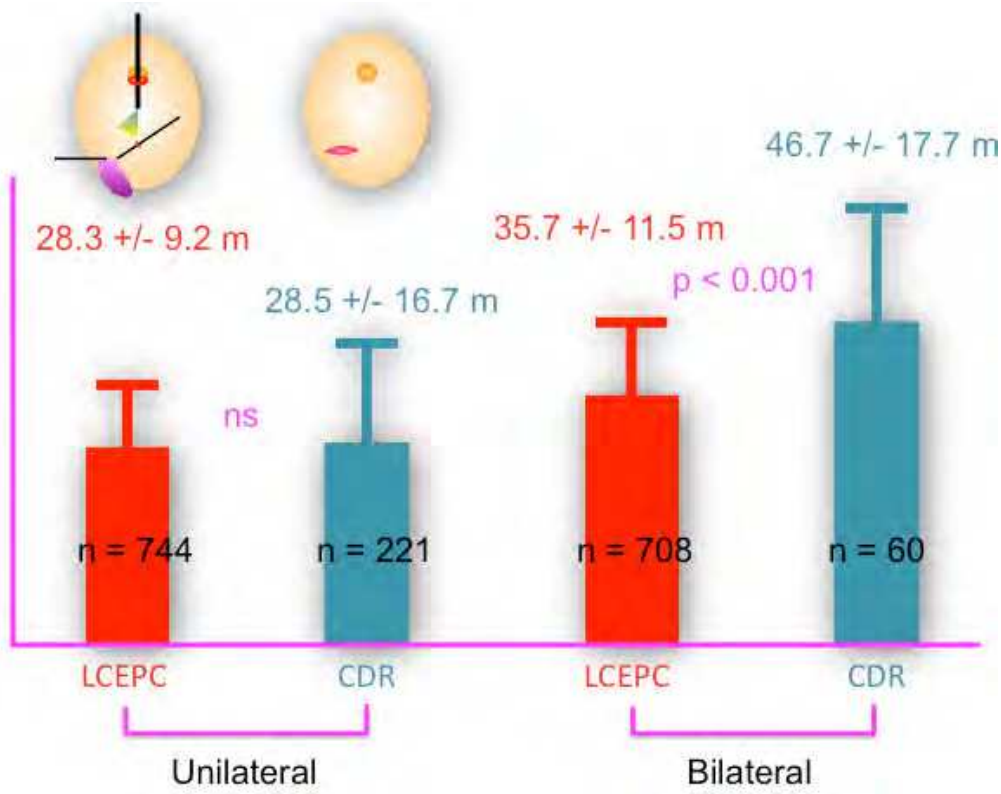

Fig. 10. Operation times in LCEPC and CDR groups. Operation times for unilateral repair were equal in both LCEPC and CDR groups (28.3 +/- $9.2 \mathrm{~m}$ for LCEPC vs. $28.5+/-16.7 \mathrm{~m}$ for CDR) and were shorter for LCEPC group in bilateral repair $(35.7+/-11.5 \mathrm{~m}$ vs. $46.7+$ /$17.7 \mathrm{~m}, \mathrm{p}<0.001)$. Patients who underwent combined operations affecting definitive herniorrhaphy were excluded from the analysis.

\begin{tabular}{|c|c|c|c|}
\hline & \\
\hline & LCEPC & CDR & Difference \\
\hline Major complication* & $\begin{array}{c}0 / 1631 \text { pts } \\
0.00 \%\end{array}$ & $\begin{array}{c}2 / 312 \mathrm{pts} \\
0.64 \%\end{array}$ & $P<0.001$ \\
\hline Contralateral hernia & $\begin{array}{c}5 / 814 \text { UCs } \\
0.61 \%\end{array}$ & $\begin{array}{c}4 / 246 \text { UCs } \\
1.63 \%\end{array}$ & n.s. \\
\hline Hernia recurrence & $\begin{array}{c}2 / 1631 \text { pts } \\
0.12 \% \\
2 / 2428 \text { PPVs } \\
0.08 \%\end{array}$ & $\begin{array}{c}3 / 312 \text { pts } \\
0.96 \% \\
3 / 378 \text { PPVs } \\
0.79 \%\end{array}$ & $\begin{array}{l}P<0.001 \\
P<0.001\end{array}$ \\
\hline
\end{tabular}

Table 1. Intraoperative and postoperative complications. Abbreviations: pts, patients; UCs, unilateral closures; n.s., not significant; PPVs, patent processus vaginalis; ${ }^{*}$, injury to ovarian duct and spermatic duct in a patient, respectively. 
[Results for second-look operations]

Among the 58 children, a total of 97 IIRs were investigated, including 36 C-IIRs in the postCDR group and $23 \mathrm{C}$-IIRs in the post-LCEPC group. The C-IIRs were classified according to the morphological findings during the operation, as follows: flat type, in which the IIR was flat and covered with thin cicatricial tissue; dimpled type, in which the IIR was covered by a dimpled scar; convergence type, in which cicatricial tissue had gathered at the center of the closed IIR; adhesion type, in which the IIR had adhesive bands from the surrounding viscera; convex type, in which the IIR bulged into the peritoneal cavity with a thick scar formation; and rod type, in which the IIR showed the rod-like protrusion of a thick scar (Fig. $11)$.

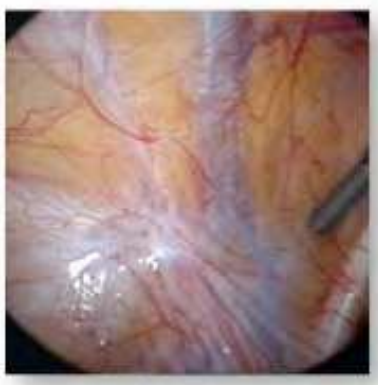

flat

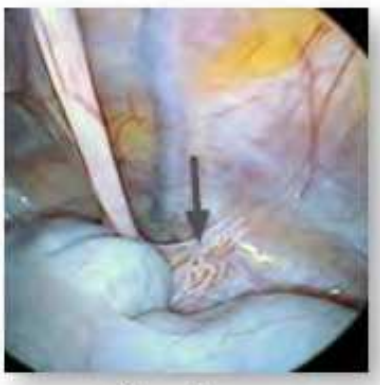

adhesion

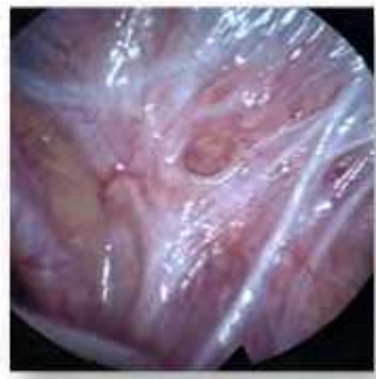

dimple

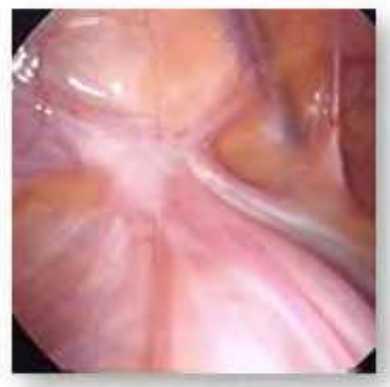

convex

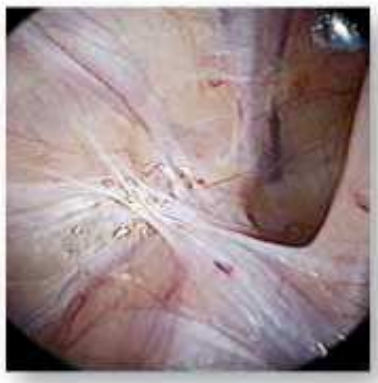

convergence

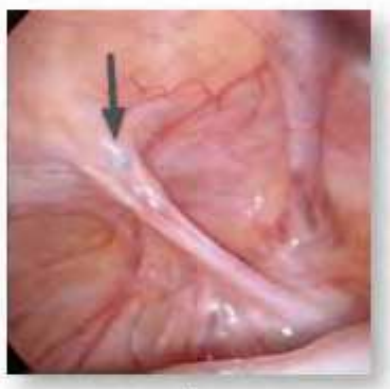

rod

Fig. 11. Spectrum of the morphological findings of closed IIRs. Type "flat", IIR is covered with thin cicatricial tissue resembling congenitally obliterated IIR; type "dimple", IIR has thick cicatricial tissue and a dimple at the center looks like recurrence of the PPV;

type "convergence", thick cicatricial tissues are gathering in the center of depressed IIR; type "adhesion", adhesive bands are seen from surrounding viscera (cecum in this example) and dislocated sutures are also seen in some cases (arrow); type "convex", IIR is covered with thick scar tissue and convex toward the intraperitoneal space; type "rod", IIR has a rod like thickening of the scar tissue protruding into the peritoneal cavity. A suture knot is seen at the closure site in this case (arrow).

The C-IIRs in the post-CDR group were mostly composed of the flat type, followed in frequency by the dimpled, convergence and adhesion types indicating that most of the 
closed IIRs were of the flat or dimpled type with/without peritoneal convergence or adhesions. On the other hand, the C-IIRs in the post-LCEPC group mostly consisted of the flat, rod or convex types, with only one case with a dimpled type, indicating that $95.7 \%$ of the closed IIRs were of a flat or convex type bulging into the peritoneal space (Fig. 12). This difference in the distribution of morphological findings was significantly different between the two groups $(\mathrm{p}<0.001)$.

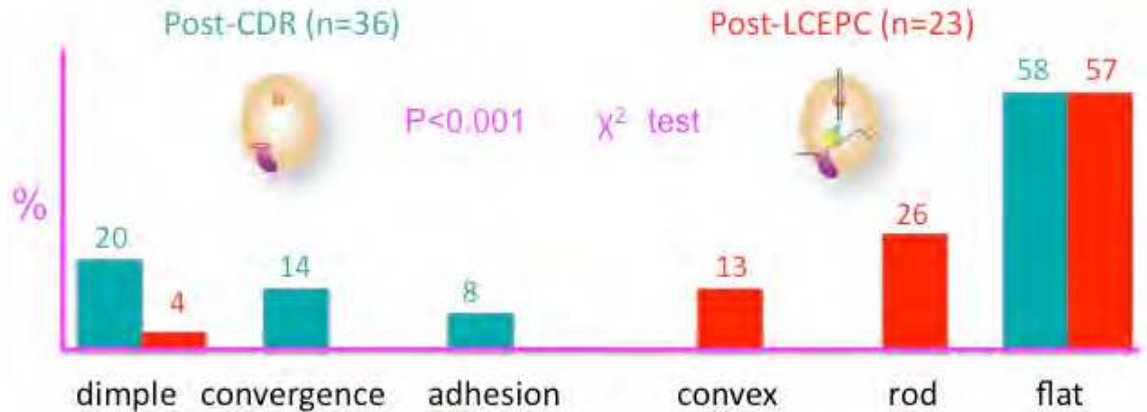

Fig. 12. Distribution of morphological appearances of the closed IIR. More than half IIRs in both groups revealed type "flat". In remaining cases, IIRs in CDR group showed types having any degenerative changes such as "dimple", "convergence" or "adhesion", while IIRs in LCEPC group had types looking like a solid foundation such as "convex" or "rod". Differences between two groups was statistically significant, $\mathrm{p}<0.001$.

\section{Discussion}

When we reported our initial experience with our technique for the laparoscopic repair of indirect inguinal hernias, some concerns existed regarding the procedure. What is the advantage of laparoscopic herniorrhaphy in children? Is it tolerable to increase the intraabdominal pressure, despite the simple closure without dividing the hernial sac? Is there any evidence that the IIR is completely closed? How can the improbability of future recurrence and the risk of adhesion be validated? Despite these questions, the primary operation sites between cases that had undergone traditional open surgery and those that have undergone laparoscopic herniorrhaphy have not been previously compared in terms of long-term outcome.

The aim of our project was based on the principle of the traditional cut-down technique involving complete extraperitoneal high ligation of the PPV and to minimize the above concerns by using a simple technique. We devised a set of needles to enable a complete circumferential ligation of the PPV using a suture that is delivered percutaneously via an extraperitoneal route in a manner that is both easy to perform and safe. The needle passes beneath the ligamentum teres uteri distal to the U-turned ovarian duct in girls, involving the ligament inside the ligature. To avoid damage to the spermatic cord and testicular vessels in boys, the needle is advanced between the peritoneum, and the cord and vessels.

At the beginning of the trial, we used some equipment that was available at that time, such as a Deschamps' needle or a biopsy needle. The key point of this technique is to pass a 
suture in and out around the IIR percutaneously under safe conditions and with precise control. After several modifications, we developed a commercial product that we named the "Endoneedle Kit". This kit can be easily used with satisfactory results and provides precise control while reducing the operation time.

Regarding the parents' perspective, the parents chose laparoscopic repair more frequently because they highly valued the cPPV closure and the cosmetic superiority, resulting in the predominance of girls and the associated morbidities of the umbilicus in the LCEPC group. The parents were very satisfied with the wound and umbilical cosmesis in the LCEPC group patients.

The average operation time for a unilateral hernia in the LCEPC group was comparable with that in the CDR group but was shorter for bilateral hernias. The technology was much easier to use in girls than in boys, as care is required around the spermatic cord. Because of the tight contact between the spermatic cord and the peritoneum, the separation of these structures in advance using electrocautery and a grasper is advisable (Endo et al., 2009), although in skilled hands, this step can be abridged.

Drawbacks associated with the reproductive system are a hidden but not negligible problem (Steigman et al., 1999, Hansen et al., 2006). We had two episodes of injury to the reproductive system during cut-down repair. On the other hand, direct intraoperative inspection of the spermatic cord and/or adnexa enables injury to these structures to be avoided.

As for postoperative complications, the incidence of metachronous hernia among children who underwent LCEPC was $0.6 \%$, which was far lower than reported incidence for traditional unilateral open repair (between 5.6\% and 30\%)( Given et al., 1989, Burd et al., 2001). Postoperative recurrence was significantly less frequent in the LCEPC group. The main factors affecting recurrence have been recognized as (1) failure to ligate the sac high enough at the internal ring, (2) injury to the floor of the inguinal canal as a result of operative trauma, (3) failure to close the internal ring in girls, and (4) postoperative wound infection and hematoma (Grosfeld et al., 1991). Our technique has proven to be a method that can avoid all these possible causes of recurrence.

To further validate the efficacy of laparoscopic closure of the PPV with regard to postoperative complications, we analyzed the laparoscopically inspected IIRs that had been closed with a definitive herniorrhaphy. A laparoscopic approach during a second-look operation enabled the correct relationships of the anatomic structures to be identified under direct vision.

Laparoscopically observed C-IIRs varied widely in appearance, ranging from a flat closed ring with scarring to somewhat protruded or depressed rings with or without cicatricial gathering, and with or without adhesive bands from the surrounding viscera. In both the post-CDR and post-LCEPC groups, approximately $50 \%$ of the children exhibited a flat closed ring without cicatricial gathering or adhesion, which was thought to indicate the unlikelihood of problems in the future. In the remaining 50\%, however, characteristic differences were found in the spectra of variations between the post-CDR and post-LCEPC groups. Most of the C-IIRs in the post-CDR group were of the flat or depressed type, while some showed cicatricial gathering or adhesions from the bladder, cecum or the omentum. In contrast, the C-IIRs in the post-LCEPC group ranged from flat to convex or the rod-like convexity type without cicatricial gathering or adhesions. This difference may have arisen from the difference in the approach to the IIR. In laparoscopic closure, the IIR is encircled by a suture proximal to the IIR level, placing all the surrounding viscera out of the IIR, while 
the cut-down procedure requires the traction of the seminal cord or round ligament from the outside so as to obtain a good visual field and achieve high ligation. The omental or intestinal loop protrusion into the operative field during closure is a common experience when the intraabdominal pressure rises because of inadequate anesthesia. A flat or convex type of IIR without any adhesions, as observed in the post-LCEPC group, may enable the maintenance of an anatomically normal IIR structure over the long-term.

\section{Conclusion}

We reported the unique technique to achieve completely extraperitoneal ligation of the PPV without any skip areas, sparing the spermatic cord and vessels under laparoscopic control, investigating the outcome, including the morphological appearance of the IIRs during second-look operations, with the outcome of traditional cut-down herniorrhaphy performed during the same period. Our technique as several advantages: the technique is easy to perform and requires a short operation time, it enables the inspection of bilateral IIRs with simultaneous closure of the $\mathrm{CPPV}$, it is associated with a minimum incidence of hernia recurrence and contralateral hernia, the reproductive systems can be left intact, the routine addition of an umbilicoplasty can be performed as needed, and the resulting wound is essentially indiscernible. The present comparative study, which included a second-look investigation of the IIRs, validated the feasibility of laparoscopic closure of the PPV, suggesting that this technique should be regarded as the gold standard for the treatment of children with an indirect inguinal hernia.

\section{Acknowledgments}

The authors gratefully acknowledge the cooperation of TSK Laboratory, Japan for developing this "Endoneedle Kit".

\section{References}

[1] Burd RS., Heffington SH., \& Teague JL. (2001), The optimal approach for management of metachronous hernias in children: a decision analysis. J Pediatr Surg, 36:1190-1995

[2] Endo M., \& Ukiyama E. (2001). Laparoscopic closure of patent processus vaginalis in girls with inguinal hernia using a specially devised suture needle. Ped Endosurg Innov Techn 5(2):187-191, 1092-6410

[3] Endo M., Watanabe T., Nakano M., Yoshida F., \& Ukiyama E (2009), Laparoscopic completely extraperitoneal repair of inguinal hernia in children: A single institute experience with 1,257 repairs compared with cut-down herniorrhaphy. Surg Endosc, 23(8):1706-1712.

[4] Given JP., \& Rubin SZ. (1989), Occurrence of contralateral inguinal hernia following unilateral repair in a pediatric hospital. J Pediatr Surg, 24:963-965

[5] Gorsler CM., \& Schier F. (2003), Laparoscopic herniorrhaphy in children. Surg Endosc, 17(4):571-573

[6] Grosfeld JL., Minnick K., Shedd F., West KW., Rescoria FJ., \& Vane DW. (1991), Inguinal hernia in children: factors affecting recurrence in 62 cases. J Pediatr Surg, 26:283-287

[7] Hansen KA., \& Eyster KM. (2006), Infertility: an unusual complication of inguinal herniorrhaphy. Fertil Steril, 86:217-218 
[8] Miltenburg DM., Nuchtern JG., Jaksic T., Kozinetiz C., \& Brandt ML. (1998), Laparoscopic evaluation of the pediatric hernia- A meta-analysis. J Pediatr Surg, 33(6):874-879

[9] Saranga BR., Arora M., \& Baskaran V. (2008), Pediatric inguinal hernia: laparoscopic versus open surgery. JSLS, 12(3): 277-281.

[10] Steigman CK., Sotelo-Avila C., \& Weber TR. (1999), The incidence of spermatic cord structures in inguinal hernia sacs from male children. Am J Surg Pathol, 23: 880-885 


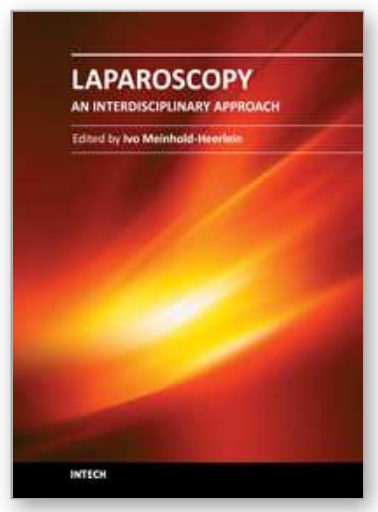

\section{Laparoscopy - An Interdisciplinary Approach}

Edited by Dr. Ivo Meinhold-Heerlein

ISBN 978-953-307-299-9

Hard cover, 146 pages

Publisher InTech

Published online 12, September, 2011

Published in print edition September, 2011

Over the last decades an enormous amount of technical advances was achieved in the field of laparoscopy. Many surgeons with surgical, urological, or gynaecological background have contributed to the improvement of this surgical approach which today has an important and fixed place in the daily routine. It is therefore comprehensible to compose a book entitled laparoscopy serving as a reference book for all three disciplines. Experts of each field have written informative chapters which give practical information about certain procedures, indication of surgery, complications and postoperative outcome. Wherever necessary, the appropriate chapter is illustrated by drawings or photographs. This book is advisable for both beginner and advanced surgeon and should find its place in the libraries of all specialties â€" surgery, urology, and gynecology.

\section{How to reference}

In order to correctly reference this scholarly work, feel free to copy and paste the following:

Masao Endo, Michinobu Ohno, Fumiko Yoshida, Miwako Nakano, Toshihiko Watanabe and Etsuji Ukiyama (2011). Laparoscopic Hernia Repair and Its Validation by Second-Look Inspection to Internal Inguinal Rings in Children with Patent Processus Vaginalis, Laparoscopy - An Interdisciplinary Approach, Dr. Ivo MeinholdHeerlein (Ed.), ISBN: 978-953-307-299-9, InTech, Available from:

http://www.intechopen.com/books/laparoscopy-an-interdisciplinary-approach/laparoscopic-hernia-repair-andits-validation-by-second-look-inspection-to-internal-inguinal-rings-i

\section{INTECH}

open science | open minds

\section{InTech Europe}

University Campus STeP Ri

Slavka Krautzeka 83/A

51000 Rijeka, Croatia

Phone: +385 (51) 770447

Fax: +385 (51) 686166

www.intechopen.com

\section{InTech China}

Unit 405, Office Block, Hotel Equatorial Shanghai

No.65, Yan An Road (West), Shanghai, 200040, China

中国上海市延安西路65号上海国际贵都大饭店办公楼405单元

Phone: +86-21-62489820

Fax: $+86-21-62489821$ 
(C) 2011 The Author(s). Licensee IntechOpen. This chapter is distributed under the terms of the Creative Commons Attribution-NonCommercialShareAlike-3.0 License, which permits use, distribution and reproduction for non-commercial purposes, provided the original is properly cited and derivative works building on this content are distributed under the same license. 